\title{
FACT - Performance of the First SiPM camera
}

D. Neise ${ }^{\star a}$, M. L. Ahnen ${ }^{a}$, A. Biland ${ }^{a}$, M. Balbo ${ }^{b}$, M. Bergmann ${ }^{c}$, T. Bretz ${ }^{a, 1}$, K. A. Brügge ${ }^{d}$, J. Buss ${ }^{d}$, D. Dorner ${ }^{c}$, S. Einecke ${ }^{d}$, J. Freiwald ${ }^{d}$, C. Hempfling ${ }^{c}$, D. Hildebrand ${ }^{a}$, G. Hughes ${ }^{a}$, W. Lustermann ${ }^{a}$, K. Mannheim $^{c}$, K. Meier $^{c}$, S. Müller ${ }^{a}$, D. Neise ${ }^{a}$, A. Neronov ${ }^{b}$, M. Nöthe ${ }^{d}$, A.-K. Overkemping ${ }^{d}$, A. Paravac ${ }^{c}$, F. Pauss $^{a}$,

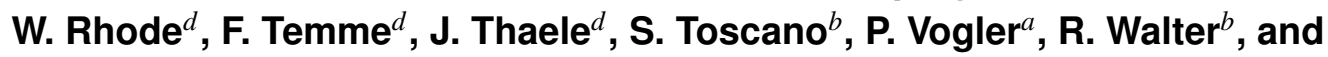
A. Wilbert ${ }^{c}$

${ }^{a}$ ETH Zurich, Institute for Particle Physics Otto-Stern-Weg 5, 8093 Zurich, Switzerland

${ }^{b}$ University of Geneva, ISDC Data Center for Astrophysics

Chemin d'Ecogia 16, 1290 Versoix, Switzerland

${ }^{c}$ Universität Würzburg, Institute for Theoretical Physics and Astrophysics

Emil-Fischer-Str. 31, 97074 Würzburg, Germany

${ }^{d}$ TU Dortmund, Experimental Physics 5 Otto-Hahn-Str. 4, 44221 Dortmund, Germany

1 also at RWTH Aachen

E-mail: neised@phys.ethz.ch

The First G-APD Cherenkov Telescope (FACT) is the first operational test of the performance of silicon photomultipliers (SiPM) in Cherenkov Astronomy. These novel photo detectors promised to be an inexpensive and robust alternative for vacuum photomultiplier tubes, but had, up to now, never been applied in an Imaging Air shower Cherenkov Telescope (IACT). For more than three years FACT has operated on La Palma, Canary Islands (Spain), with the primary aim of long-term monitoring of astrophysical sources.

Stable performance of the photo detectors is crucial and therefore has been studied in great detail. Special care has been taken with regards to their temperature and over voltage dependence through implementation of a feedback method for keeping their properties stable. Several independent long term measurements were conducted to analyze and verify SiPM gain stability. Dark count spectra, which also make for an excellent self calibration mechanism, were used to study and correct for temperature dependencies. Rate scans make it possible to derive a method, for quickly finding appropriate trigger thresholds by measuring pixel currents, and thus allow for a consistent data acquisition rate. Dedicated measurements using an LED flasher are used to study the correct application of SiPM bias voltages.

In this paper, the results of the long term studies will be presented and the applicability of SiPMs in IACTs for long term monitoring will be shown.

The 34th International Cosmic Ray Conference,

30 July- 6 August, 2015

The Hague, The Netherlands

* Speaker. 


\section{Introduction}

Since November 2011 the First G-APD Cherenkov Telescope (FACT) has operated on the Roque de los Muchachos on the Canary Island La Palma. The camera is equipped with 1440 arrays of avalanche photodiodes operated in Geiger mode (G-APD) commonly referred to as SiPMs (Hamamatsu MPPC S10362-33-50C). With a focal length of about $5 \mathrm{~m}$ and a mirror area of $10 \mathrm{~m}$, the instrument is comparable to the planned CTA SST designs [1]. The field of view of the camera is $4.5^{\circ}$.

One of the goals of FACT was to study the usability of these novel photo sensors as part of an exposed Cherenkov telescope camera. Another goal is longterm monitoring of bright AGNs [3].

SiPMs are mechanically and electronically robust, do not age under strong illumination and do not need high voltages to operate, they provide a gain on the order of $10^{5}$ to $10^{6}$, good intrinsic time resolution and a photo detection efficiency better than common photo multiplier tubes (PMT). Possible challenges are the temperature dependence of detector characteristics such as the gain, but also spuriously generated discharges called dark count events and so called optical cross talk [6].

\section{Keeping SiPM Gain Stable}

A homogeneous photo-sensor gain is important for the operation of an Imaging Air Cherenkov Telescope (IACT) camera. Temporal stability of the gain is important to keep the trigger response is stable over time.

Different methods are available to determine the SiPM gain. Utilizing so called dark count signal spectra has proven to be suitable for FACT. In order to generate a dark count signal spectrum, the spurious discharges generated by an SiPM in the absence of light are analyzed. The signal extraction method is depicted in Figure 1.

Due to an effect known as optical crosstalk [6], a single avalanche in a G-APD can trigger additional avalanche discharges in neighboring G-APDs. An example result for dark count spectrum of a randomly chosen pixel is shown in Figure 2. The gain is extracted as the distance of two consecutive peaks in the obtained spectrum.

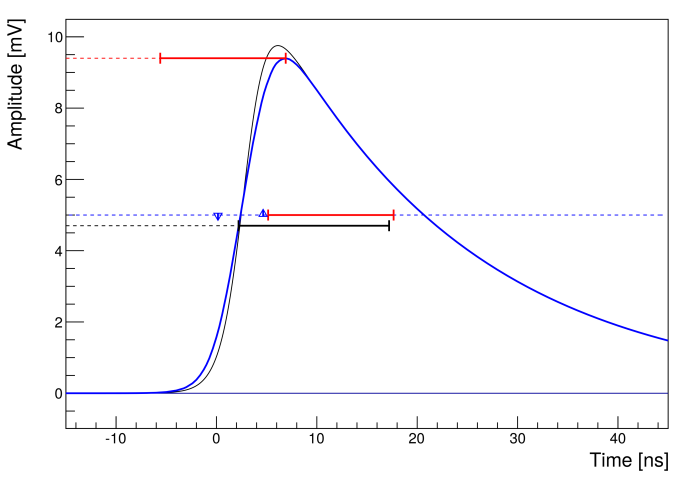

Figure 1: The sampled signal (black) is smoothed by a sliding average filter (blue). If a local maximum is found after the leading edge (within lower red time window), the pulse qualifies for integration (30 samples, black time window). From [4].

The gain $M$ of the SiPMs only depends on the over-voltage $\Delta U$ and can be described as

$$
M(T, U) \propto \Delta U=U-U_{b r}(T) .
$$

Where $U$ denotes the bias voltage applied to the SiPM, $T$ is the SiPM temperature, and $U_{b r}$ is the sensor specific breakdown voltage which is linearly depending on the temperature with $\partial U_{b r} / \partial T=55 \mathrm{mV} / \mathrm{K}$. 
A total of 28 temperature sensors have been distributed inside the photo-sensor plane, in order to measure its temperature profile. The temperature of each group of SiPMs being supplied by the same bias voltage line is determined by interpolation [4]. During the observation, the temperature obtained is used to counteract any temperature changes of the SiPMs, e.g. caused by ambient air temperature.

When using passive bias supply distribution networks and filters, as in case of FACT, variations in the photo-sensors bias current consumptions will affect the bias voltage seen by the SiPM. Changing ambient light conditions, will therefor alter the effective SiPM bias voltage. For this reason a temperature stabilization of SiPMs is not sufficient to keep their over-voltage stable, but adjustable power supplies are needed.

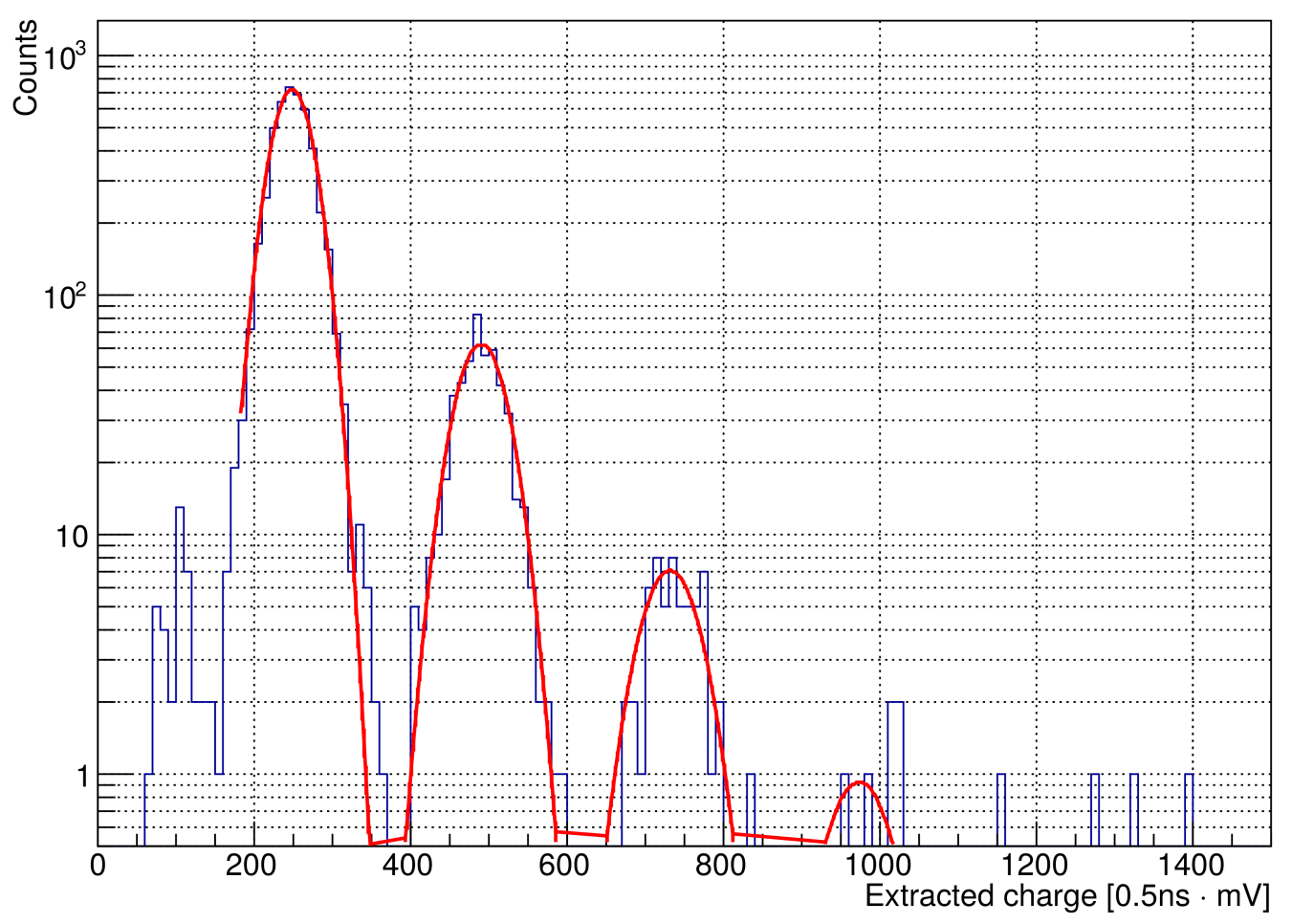

Figure 2: Example dark count spectrum of a typical FACT SiPM. The gain is defined as the distance of neighboring peaks. From [4].

In Figure 3 the stability of the gain at different sensor temperatures can be observed. As dark count spectra can currently only be obtained with FACT, when the camera lid is closed, this measurement is unsuitable to show the stability of the gain also during observation.

In order to study the gain stability under changing ambient light conditions also with an open camera lid an external calibration device was used.

An LED flasher device can illuminate the FACT camera homogeneously. Care has been taken to stabilize the light yield of the LEDs with respect to the ambient temperature by means of an optical feedback driver [2].

The FACT camera is triggering on these calibration light flashes, with an increased trigger threshold. In Figure 4, the average light pulser signal for different ambient light conditions can be 


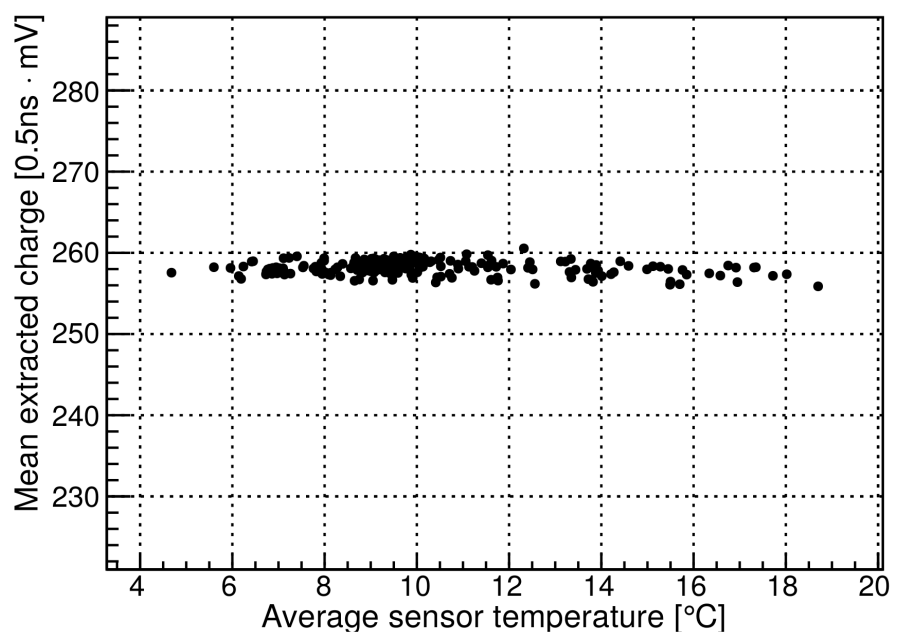

Figure 3: Extracted charge from dark count spectra at different temperatures. From [4].

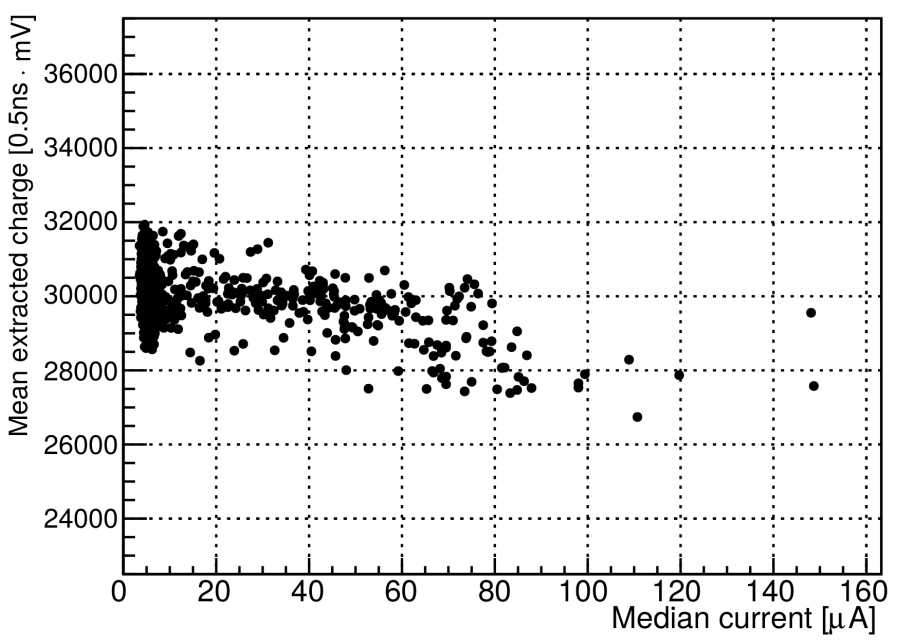

Figure 4: Extracted charge vs. median current as a measure of the ambient light.Mainly stable gain under varying ambient light conditions. The residual is in the order of 5\%. From [4].

seen. Under dark night conditions the typical SiPM current is on the order of $5 \mu \mathrm{A}$.

A small residual effect on the ambient light conditions is still visible and might be due to a biased SiPM temperature measurement. While under dark night conditions the SiPM temperature is mainly determined by the ambient temperature, waste heat of the SiPMs under moonlight conditions $(\approx 10 \mathrm{~W}$ dissipated by the SiPMs) may create local hot spots which are not very well resolved by the temperature sensors.

\section{Predicting the trigger threshold}

The trigger threshold needs to be adjusted for changes in the ambient light conditions. As seen in Figure 5, the nominal trigger threshold, varies dramatically when ambient light conditions change. The maximum trigger rate of the FACT data acquisition system is $250 \mathrm{~Hz}$. In order to keep the dead 


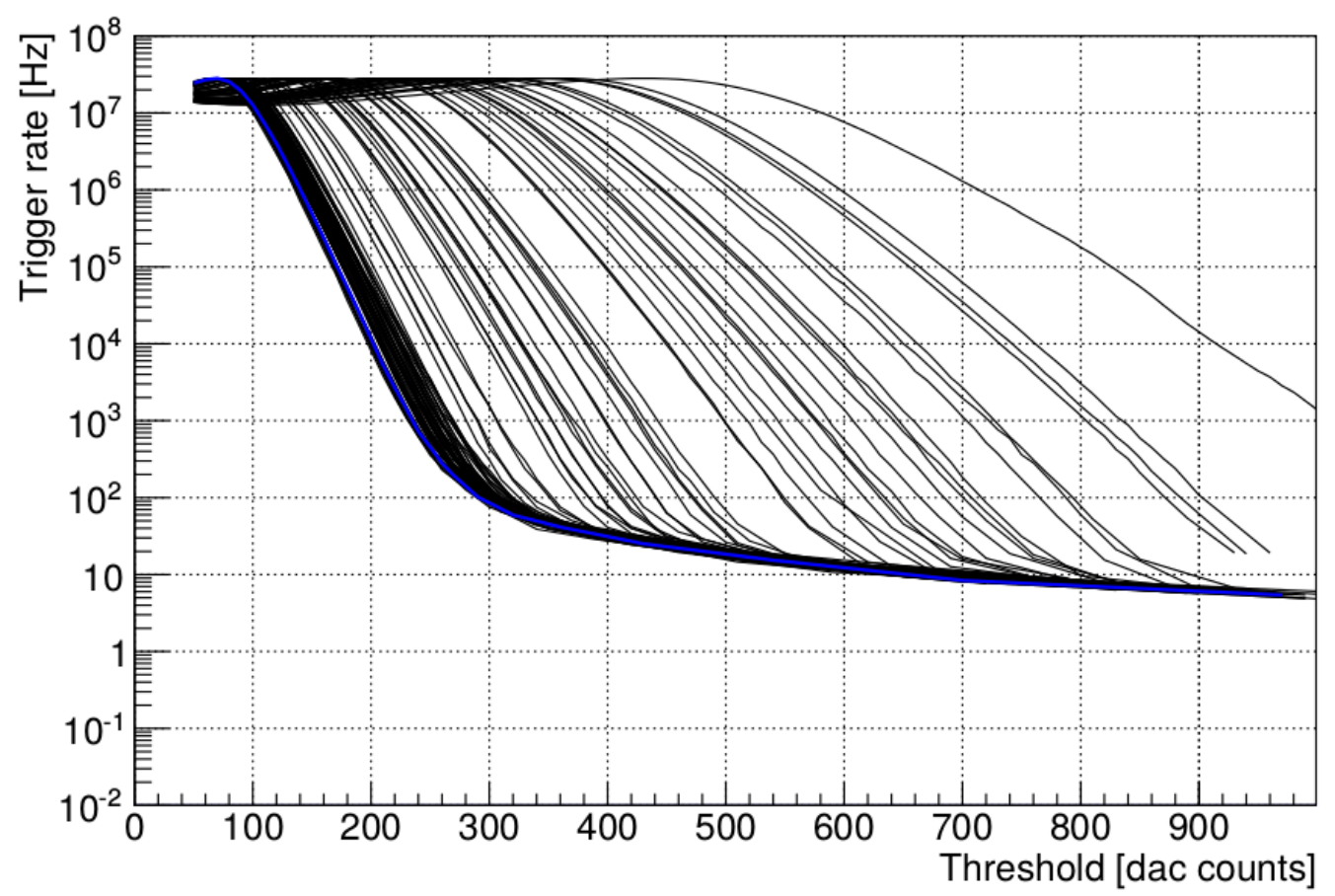

Figure 5: Trigger rates for different ambient light conditions versus trigger threshold, so called rate scans. The blue curve was taken during dark night and clear sky. The black curves were taken during different moon phases. From [4].

time lower than $5 \%$, the nominal trigger frequency is aimed to be $60 \mathrm{~Hz}$ which results in a dead time of $3.3 \%$. The trigger threshold is kept constant during an entire run (typically $5 \mathrm{~min}$ ) in order to provide stable observation conditions. As the photo-sensor gain is kept exceptionally stable, it was possible to find a description for a suitable trigger threshold, which solely depends on the average SiPM current. Thus, there is no need to conduct long lasting scans to establish a suitable trigger threshold for a given run. The relationship between a suitable trigger threshold $t$ and the average SiPM current $I$ can be described by

$$
t=(156.3 \pm 1.2)\left(\frac{I}{\mu \mathrm{A}}\right)^{0.3925 \pm 0.0022} .
$$

This is shown in Figure 6. The lowest trigger thresholds normally used during clear new moon nights are around 280 DAC units, which roughly corresponds to 20 p.e. per trigger patch summing the analog signals of nine pixels.

\section{Current prediction}

Reliable determination of data quality is necessary for a monitoring instrument such as FACT. Predicting the ambient light level from first principles, allows to compare the actually measured SiPM current to the predicted current. Deviations from the prediction can be interpreted as adverse observation conditions. 


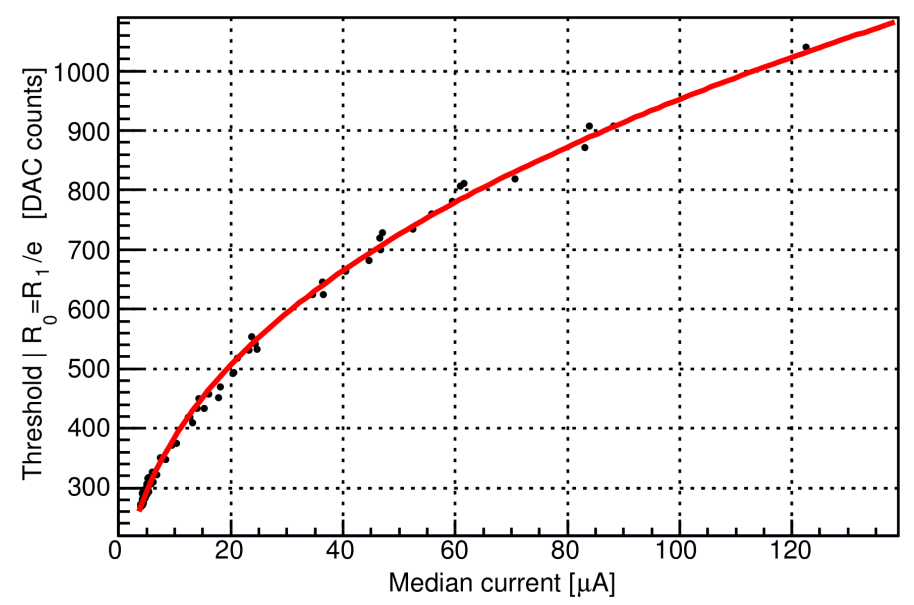

Figure 6: Trigger threshold prediction for arbitrary ambient light conditions. The red line is the trigger threshold prediction from Equation 3.1. From [4].

Likewise, the trigger threshold and thus the energy threshold depend on the ambient light, so by predicting it, one can improve the observation schedule.

An empirical formula is used, which describes the average SiPM current as a function of the suns zenith distance, the moon phase, the moons altitude and angular separation from the line of sight, cf. [5].

\section{Understanding SiPM cross talk}

Proper detector simulations are required for precise primary particle energy reconstruction in case of IACT. In order to simulate the photo-sensors correctly a well description of SiPM properties is important. Due to the high quality of gain determination using dark count spectra, it was possible to create a dark spectrum with very high statistics summing over the dark spectra of all camera pixels. This sum spectrum could not be fitted well with a geometrical or Borel distribution. The sum spectrum shown in Figure 7 was fitted by a modified Erlang distribution, which in turn was used in the Monte Carlo simulation of FACT.

\section{Conclusion}

We have shown that the devised method of gain stabilization is able to keep the average SiPM gain variations smaller than $0.5 \%$. The gain homogeneity over the entire camera plane varies by less than $2.5 \%$. Additionally, the detector simulation was improved by finding a suitable description of SiPM dark counts. 


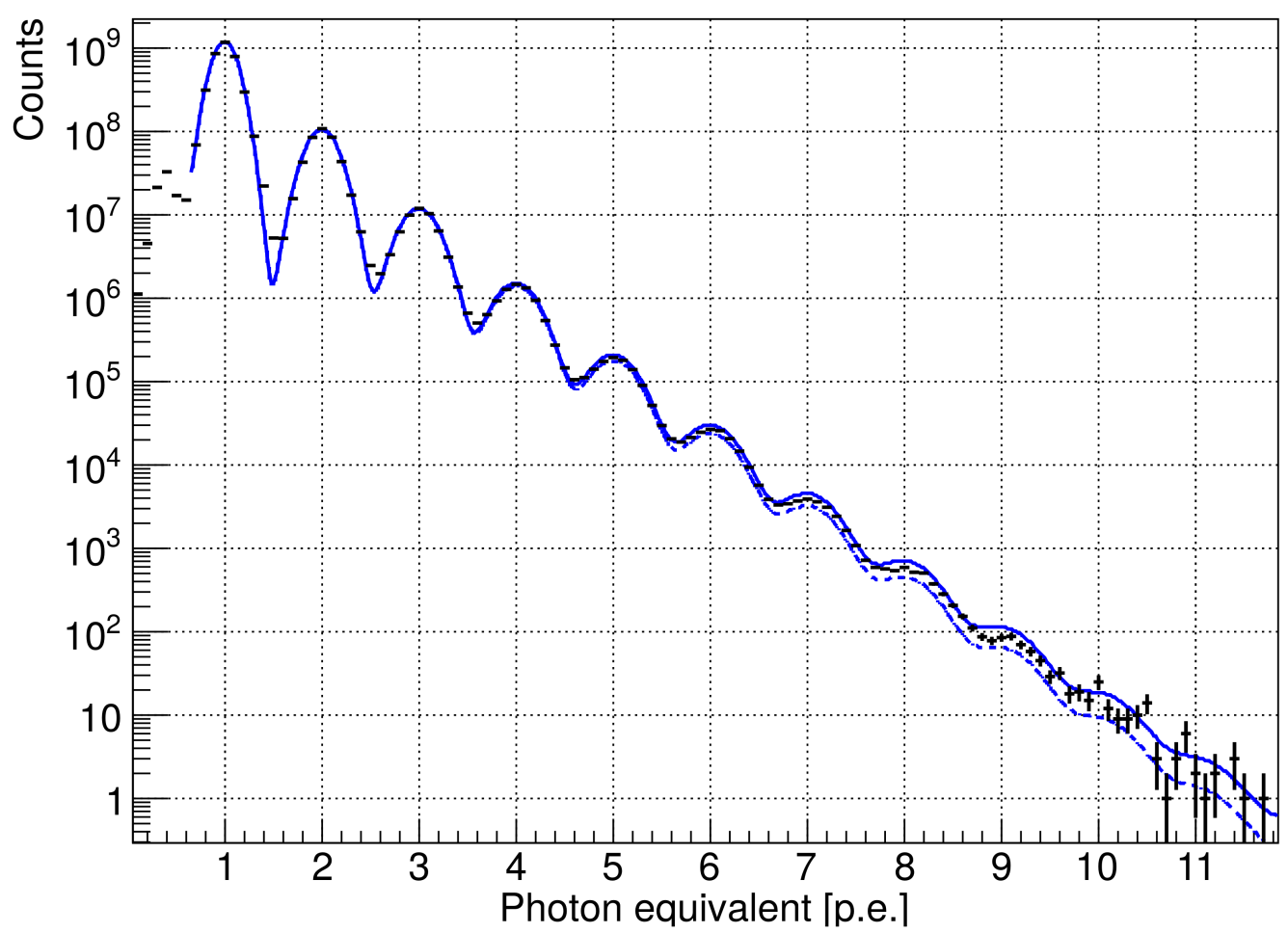

Figure 7: Sum of normalized dark count spectra of all pixels. Each individual dark count spectrum has been normalized to its respective gain before summation. The dashed blue line is a fit with a modified Erlang distribution. The solid blue line is a fit with a standard Erlang distribution. An Excellent description of SiPM crosstalk has been achieved thanks to a well understood gain. From [4]. 
Acknowledgments The important contributions from ETH Zurich grants ETH-10.08-2 and ETH-27.12-1 as well as the funding by the German BMBF (Verbundforschung Astro- und Astroteilchenphysik) and HAP (Helmoltz Alliance for Astroparticle Physics) are gratefully acknowledged. We are thankful for the very valuable contributions from E. Lorenz, D. Renker and G. Viertel during the early phase of the project. We thank the Instituto de Astrofisica de Canarias allowing us to operate the telescope at the Observatorio del Roque de los Muchachos in La Palma, the Max-Planck-Institut für Physik for providing us with the mount of the former HEGRA CT 3 telescope, and the MAGIC collaboration for their support.

\section{References}

[1] M. Actis. Design concepts for the Cherenkov Telescope Array CTA: An advanced facility for ground-based high-energy gamma-ray astronomy. In: Experimental Astronomy 32.3 (2011), pp. 193-316. ISSN: 09226435. DOI: 10 . 1007/s10686-011-9247-0. arXiv: 1008 . $3703 v 3$.

[2] H. Anderhub. Design and operation of FACT - the first G-APD Cherenkov telescope. In: JINST 8.06 (June 2013), P06008. ISSN: 1748-0221. DOI: 10 . $1088 / 1748-0221$ / 8/0 6/P 06008.

[3] H. Anderhub. FACT - the First Cherenkov Telescope using a G-APD Camera for TeV Gammaray Astronomy (HEAD 2010). In: Nucl Instrum Meth A 639.1 (2010), pp. 1-4.

[4] A. Biland. Calibration and performance of the photon sensor response of FACT - The First GAPD Cherenkov telescope. In: JINST 10012 (2014). ISSN: 17480221. DOI: $10.1088 / 1748-$ $0221 / 9 / 10 / \mathrm{P} 10012$. arXiv: 1403.5747.

[5] M. L. Knoetig et al. FACT - Long-term stability and observations during strong Moon light. In: Proceedings of the 33rd ICRC (2013). arXiv: 1307 . 6116v1.

[6] D. Renker. Geiger-mode avalanche photodiodes, history, properties and problems. In: Nucl Instrum Meth A 567.1 (Nov. 2006), pp. 48-56. ISSN: 01689002. DOI: $10.1016 / \mathrm{j}$. nima . 2006.05 .060 . 